\title{
An Improved Local Gradient Method for Sea Surface Wind Direction Retrieval from SAR Imagery
}

\author{
Lizhang Zhou ${ }^{1}$ (D), Gang Zheng ${ }^{1, *}$, Xiaofeng Li ${ }^{2}$ (D), Jingsong Yang ${ }^{1}$, Lin Ren ${ }^{1}$, Peng Chen ${ }^{1}$, \\ Huaguo Zhang ${ }^{1}$ and Xiulin Lou ${ }^{1}$ \\ 1 State Key Laboratory of Satellite Ocean Environment Dynamics, Second Institute of Oceanography, \\ State Oceanic Administration, Hangzhou 310012, China; zlz19921122@163.com (L.Z.); \\ jsyang@sio.org.cn (J.Y.); renlin210@sio.org.cn (L.R.); chenpeng@sio.org.cn (P.C.); zhanghg@sio.org.cn (H.Z.); \\ lxl@sio.org.cn (X.L.) \\ 2 GST at National Oceanic and Atmospheric Administration (NOAA)-National Environmental Satellite, \\ Data, and Information Service (NESDIS), College Park, MD 20740, USA; xiaofeng.li@noaa.gov \\ * Correspondence: zhenggang@sio.org.cn
}

Received: 21 April 2017; Accepted: 26 June 2017; Published: 30 June 2017

\begin{abstract}
Sea surface wind affects the fluxes of energy, mass and momentum between the atmosphere and ocean, and therefore regional and global weather and climate. With various satellite microwave sensors, sea surface wind can be measured with large spatial coverage in almost all-weather conditions, day or night. Like any other remote sensing measurements, sea surface wind measurement is also indirect. Therefore, it is important to develop appropriate wind speed and direction retrieval models for different types of microwave instruments. In this paper, a new sea surface wind direction retrieval method from synthetic aperture radar (SAR) imagery is developed. In the method, local gradients are computed in frequency domain by combining the operation of smoothing and computing local gradients in one step to simplify the process and avoid the difference approximation. This improved local gradients (ILG) method is compared with the traditional two-dimensional fast Fourier transform (2D FFT) method and local gradients (LG) method, using interpolating wind directions from the European Centre for Medium-Range Weather Forecast (ECMWF) reanalysis data and the Cross-Calibrated Multi-Platform (CCMP) wind vector product. The sensitivities to the salt-and-pepper noise, the additive noise and the multiplicative noise are analyzed. The ILG method shows a better performance of retrieval wind directions than the other two methods.
\end{abstract}

Keywords: local gradients method; retrieval; SAR; wind direction

\section{Introduction}

Surface wind field over the oceans is needed for weather forecasts, wind resources assessment, numerical modeling of waves, oil spill monitoring, and so on [1-10]. Traditional measurements of sea surface wind from ships, buoys and land stations are far from meeting the growing demand for human beings, as these approaches can only provide data with limited spatial and temporal coverage. Spaceborne microwave radiometers and scatterometers have provided global-scale observations for sea surface wind, but the wind data in coastal regions are missing due to contamination from signal reflection of land [11-13]. Also, the spatial resolution of radiometers or scatterometers is relatively low (about $25 \mathrm{~km}$ ). This is suitable for open ocean studies. However, wind fields on a much finer scale can be provided by spaceborne synthetic aperture radar (SAR) due to its relatively high spatial resolution. As a result, SARs can be useful tools when high-resolution ocean surface wind fields are needed, especially in coastal regions, and the retrieval of wind field from SAR images is widely researched [14-21]. 
Many satellites have been launched with SAR onboard, e.g., Seasat, ERS-1/2, Envisat, Radarsat-1/2, and so on [14-18]. These sensors have acquired abundant SAR images containing many interesting features including coastal upwelling, typhoon/hurricane, atmospheric waves, atmospheric vortex street, and so on [19-31]. Some of SAR imaged features, e.g., Langmuir cells, boundary layer rolls, surfactant streaks, foam and water blown from breaking waves, or wind shadowing, align with wind direction.

Wind direction (with an ambiguity of $180^{\circ}$ ) can be retrieved from these features by different methods including Fourier transforms, wavelet analysis, local gradients and so on. The $180^{\circ}$ ambiguity can be removed by referencing to weather model output, Doppler shift or land shadows [3,16,32]. The accuracy of various methods ranges from $15-40^{\circ}$ [33]. After wind direction is obtained, wind speed can be retrieved by physical or empirical models. Accordingly, estimating wind vectors directly from SAR images becomes feasible [34]. Both cross-polarization and co-polarization SAR images can be used for the retrieval of wind vectors [35-37]. There are two conventional methods of retrieving wind directions (with an ambiguity of $180^{\circ}$ which is removed later) from SAR images, namely, two-dimensional fast Fourier transform (2D FFT) method and local gradients (LG) method [34,38,39]. In the FFT method, the Fourier spectrum of SAR images is computed and the main spectral energy is located perpendicular to the orientation of the wind streaks. The reported standard deviations of FFT method are between $10-37^{\circ}$ and the method works fine on large image areas, e.g., $20 \mathrm{~km}$ by $20 \mathrm{~km}$ [34]. In the LG method, local gradients are computed with standard image processing algorithms, and the orthogonal of the most frequent gradient direction is chosen to be the likely wind direction. The reported directional error of LG method was about $20^{\circ}$ for ERS- $1 / 2$ images and the most frequent spatial sampling used was $20 \mathrm{~km}$ by $20 \mathrm{~km}$ and $10 \mathrm{~km}$ by $10 \mathrm{~km}$ [34].

The tests in [34] indicated that the LG method could provide a higher resolution of retrieved wind field than the FFT method. However, the current LG method has a problem; that is, the local gradients in the conventional LG method are computed with difference approximation like Sobel operators, and this process is easily affected by noise, e.g., speckle noise. Thus, SAR images are usually first smoothed before computing local gradients. In this study, we develop an improved local gradients (ILG) method for sea surface wind retrieval by combining smoothing and computation of the local gradients together in the frequency domain. In the method, the computation of local gradients is analytical as the Gaussian function can be expressed analytically in both spatial domain and frequency domain and it can avoid the errors of difference approximation which can be easily affected by noise. The new method is tested on the images acquired by the advanced synthetic aperture radar (ASAR) onboard Envisat, and its retrieved results show better agreement with both following kinds of interpolating wind directions than the other two methods. The interpolating wind directions from the European Centre for Medium-Range Weather Forecast (ECMWF) reanalysis data are obtained by interpolating the ECMWF reanalysis data to the SAR imaging times. The same procedure is applied to the Cross-Calibrated Multi-Platform (CCMP) data to obtain the interpolating wind directions from the CCMP data.

The remaining sections are organized as follows. In Section 2, the ILG method is described in detail. In Section 3, the data sets used are introduced. In Section 4, we compare the three wind direction retrieval methods using the wind directions from the ECMWF reanalysis data and the CCMP wind products. The ILG method is also tested by using small images (thus high resolution) in this section. The performance of each retrieval method is analyzed while the images are corrupted by different types of noise in Section 5. Conclusions are given in Section 6.

\section{Improved Local Gradients Method}

The direction of the gradient should be the same as the direction of the strongest change in an image, and an ideal image of streaks should have nearly no change along the direction of streaks, and show the strongest variation in the orthogonal direction of streaks. Thus, the wind direction, which is assumed to be parallel to the wind streaks [40], is also perpendicular to the direction of the gradient. 
Usually, the components of the gradient are computed with the optimized Sobel operators pixel by pixel after smoothing, and the most frequent gradient direction is chosen to be the local wind direction.

As mentioned in the introduction, the computing method described above, however, can lead to some problems. So, we try to combine the operation of smoothing and computation of local gradients on the basis of a conventional LG method which is given by Koch in 2004. The smoothing of an image can be realized by convolution, and the Gaussian function is commonly used for smoothing, as shown in

$$
s^{\prime}\left(x^{\prime}, y^{\prime}\right)=\iint s(x, y) f\left(x-x^{\prime}, y-y^{\prime}\right) d x d y
$$

where $s^{\prime}\left(x^{\prime}, y^{\prime}\right)$ is the image after smoothing, and $s(x, y)$ is the original image. $f(\cdot, \cdot)$ is the Gaussian function for smoothing, with the form

$$
f(x, y)=\frac{1}{2 \pi \sigma^{2}} e^{-\frac{x^{2}+y^{2}}{2 \sigma^{2}}}
$$

where $\sigma$ determines the width of smoothing window in the ILG method. The choice of the value of the parameter is empirical, and in this paper the parameter is set to 15 for all SAR images. The benefit of the choice of the Gaussian is that it can be expressed analytically in both spatial and frequency domains. The gradients of the image can be expressed as

$$
\nabla s^{\prime}\left(x^{\prime}, y^{\prime}\right)=\frac{\partial}{\partial x^{\prime}} s^{\prime}\left(x^{\prime}, y^{\prime}\right) \hat{\mathrm{e}}_{x}+\frac{\partial}{\partial y^{\prime}} s^{\prime}\left(x^{\prime}, y^{\prime}\right) \hat{\mathrm{e}}_{y}
$$

where $\hat{\mathrm{e}}_{x}$ and $\hat{\mathrm{e}}_{y}$ are the unit vectors in the $x$ and $y$ directions, respectively. Using Equation (1)-(3) and exchanging the order of integral and partial derivative, $\frac{\partial}{\partial x^{\prime}} s^{\prime}\left(x^{\prime}, y^{\prime}\right)$ can be expressed as

$$
\frac{\partial}{\partial x^{\prime}} s^{\prime}\left(x^{\prime}, y^{\prime}\right)=-s(x, y) * h_{x}(x, y)
$$

where * denotes convolution, and $h_{x}(x, y)=\frac{\partial}{\partial x} f(x, y)$. We perform Fourier transform on both sides of Equation (4), and get

$$
F\left[\frac{\partial}{\partial x^{\prime}} s^{\prime}\left(x^{\prime}, y^{\prime}\right)\right]=-F[s(x, y)] \cdot F\left[h_{x}(x, y)\right]
$$

where $F[\cdot]$ denotes the 2D Fourier transform.

The first term on the right hand side of Equation (5) can be obtained from the Fourier transform of the image directly. The second term, represented by $H_{x}$ for convenience of expression, can be expressed as

$$
H_{x}=F\left[h_{x}(x, y)\right]=-\frac{i k_{x}}{\sigma^{2}} e^{-2 \sigma^{2} \pi^{2}\left(k_{x}^{2}+k_{y}^{2}\right)}
$$

where $i$ indicates the imaginary part. So, $\frac{\partial}{\partial x^{\prime}} s^{\prime}\left(x^{\prime}, y^{\prime}\right)$ can be expressed as

$$
\frac{\partial}{\partial x^{\prime}} s^{\prime}\left(x^{\prime}, y^{\prime}\right)=-F^{-1}\left\{F[s(x, y)] \cdot H_{x}\right\}
$$

where $F^{-1}[\cdot]$ denotes the 2D inverse Fast Fourier transform (2D IFFT). Similarly, $\frac{\partial}{\partial y^{\prime}} s^{\prime}\left(x^{\prime}, y^{\prime}\right)$ can be expressed as

$$
\frac{\partial}{\partial y^{\prime}} s^{\prime}\left(x^{\prime}, y^{\prime}\right)=-F^{-1}\left\{F[s(x, y)] \cdot H_{y}\right\}
$$

where $H_{y}$ can be expressed by Equation (9).

$$
H_{y}=F\left[h_{y}(x, y)\right]=-\frac{i k_{y}}{\sigma^{2}} e^{-2 \sigma^{2} \pi^{2}\left(k_{x}^{2}+k_{y}^{2}\right)}
$$


$\frac{\partial}{\partial x^{\prime}} s^{\prime}\left(x^{\prime}, y^{\prime}\right)$ and $\frac{\partial}{\partial y^{\prime}} s^{\prime}\left(x^{\prime}, y^{\prime}\right)$ are denoted as $g_{x}$ and $g_{y}$, respectively, for convenience, so the gradients can be stored as complex numbers in the form of

$$
g=g_{x}+i g_{y}
$$

Now, the gradients are in the same form as those in Koch's LG method [34]. Figure 1 shows the flowchart of the ILG method for wind direction retrieval. The remaining steps are consistent with those in Koch's method, including discarding unusable points and extracting the main gradient direction. The $180^{\circ}$ wind direction ambiguity is removed by referencing coincident wind direction from the ECMWF model result [41].

The image acquired by the advanced synthetic aperture radar (ASAR) onboard Envisat at 02:06 on 14 October 2007 is shown in Figure 2a. Figure $2 b$ shows the wind direction computed on a $30 \mathrm{~km}$ by $30 \mathrm{~km}$ subimage which is indicated by red box in Figure 2a. Wind streaks are visible all over the subimage. The white arrow $\left(211^{\circ}\right)$ in Figure $2 \mathrm{~b}$ indicates the wind direction computed from $75 \mathrm{~m}$ pixels by using the ILG method. The wind direction from the ECMWF was $222^{\circ}$, indicated by the red arrow in Figure $2 b$. The wind direction from the CCMP was $225^{\circ}$ (the green arrow in Figure $2 b$ ). Note that the wind directions here are placed in the same coordinate. In this coordinate, the direction of the northward wind vector is $0^{\circ}\left(\right.$ or $\left.360^{\circ}\right)$, the direction of the eastward wind vector is $90^{\circ}$, the direction of the southward wind vector is $180^{\circ}$, and the direction of the westward wind vector is $270^{\circ}$.

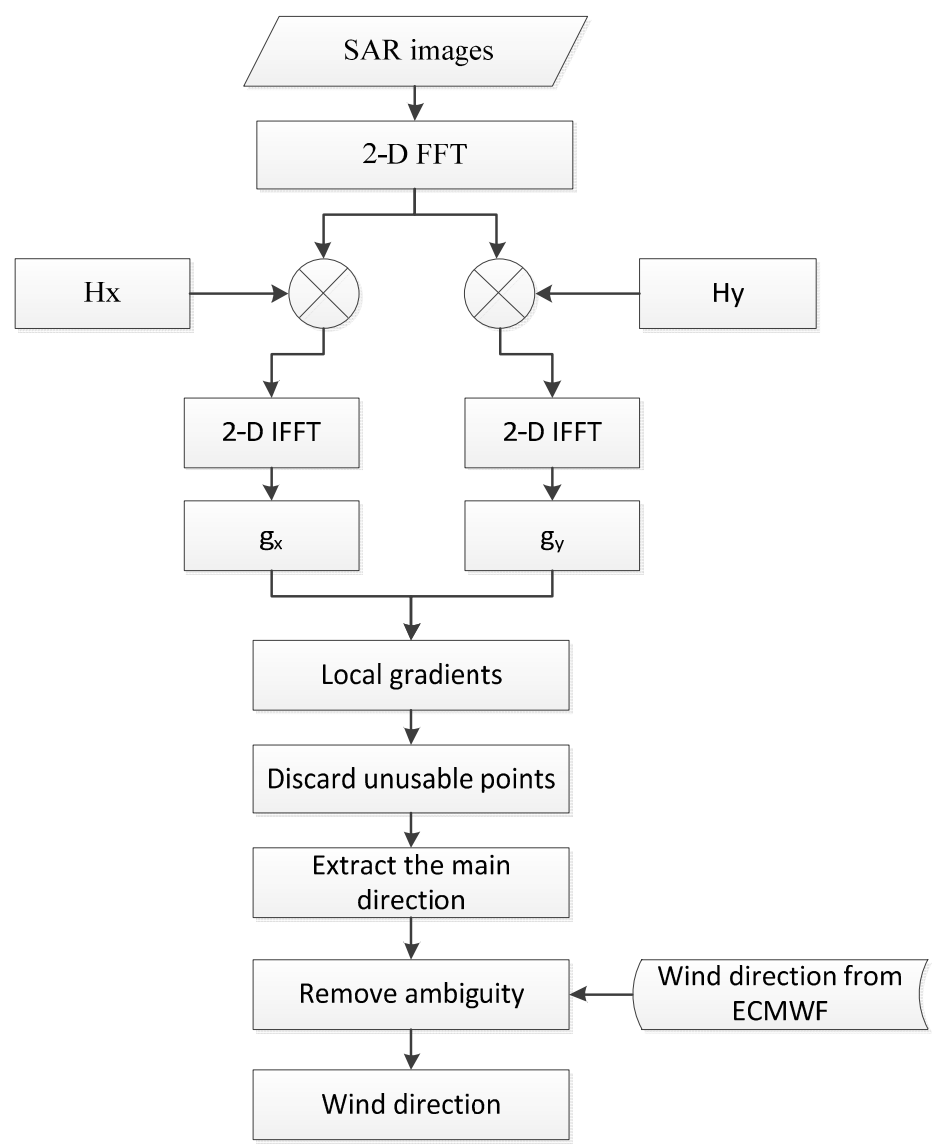

Figure 1. Flowchart of the improved local gradients method for wind direction retrieval. 


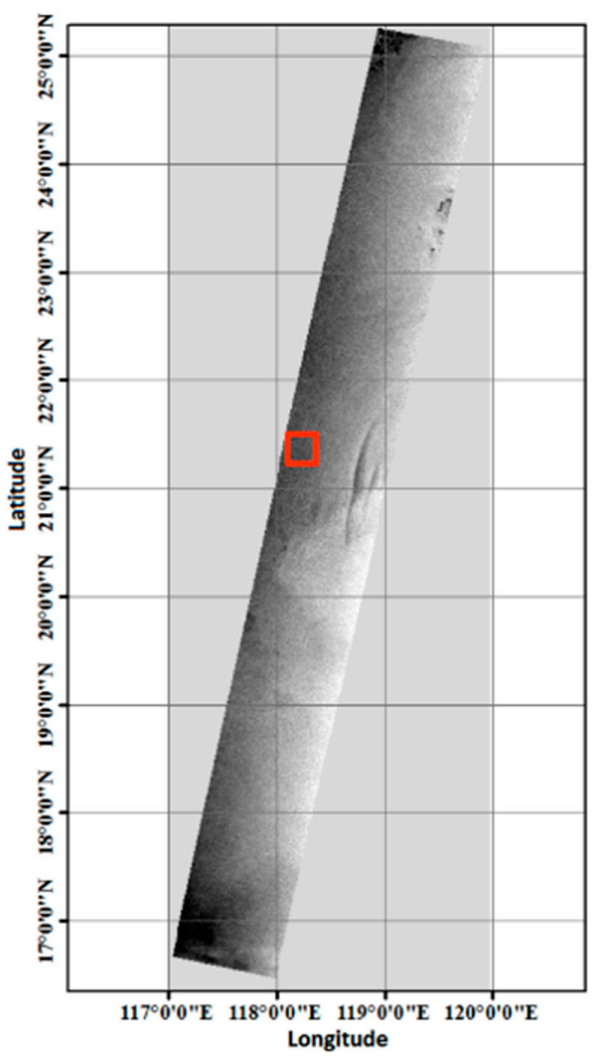

(a)

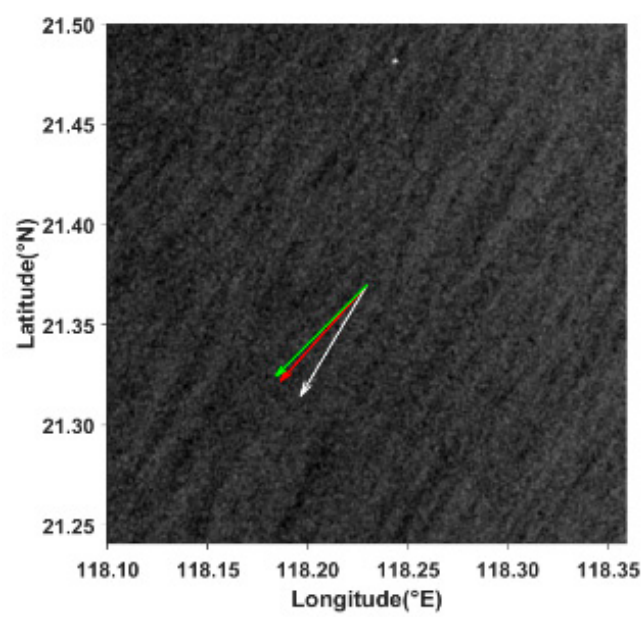

(b)

Figure 2. (a) An Envisat advanced synthetic aperture radar (ASAR) image acquired at 02:06 on 14 October 2007 in VV polarization with a resolution of $75 \mathrm{~m}$. (b) The subimage which is indicated by the red box in (a). White, red and green arrows indicate wind directions obtained from improved local gradients (ILG) method, European Centre for Medium-Range Weather Forecast (ECMWF) and Cross-Calibrated Multi-Platform (CCMP), respectively.

$H_{x}$ and $H_{y}$ are shown in Figure 3 in frequency domain, and their amplitudes are indicated by different colors. The computed $g_{x}$ and $g_{y}$ of the image in Figure $2 \mathrm{~b}$ are shown in Figure 4 in spatial domain and different colors indicate different values of the gradient component.
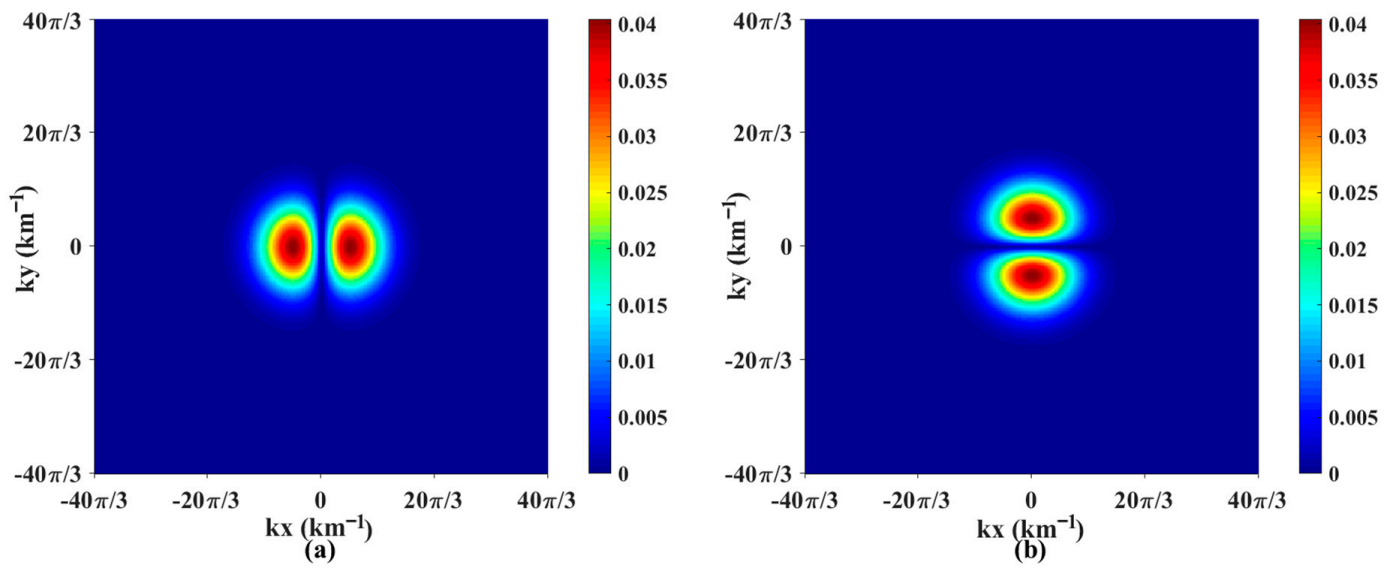

Figure 3. The amplitude distributions of $H_{x}(\mathbf{a})$ and $H_{y}(\mathbf{b}), \sigma$ is 15 . 

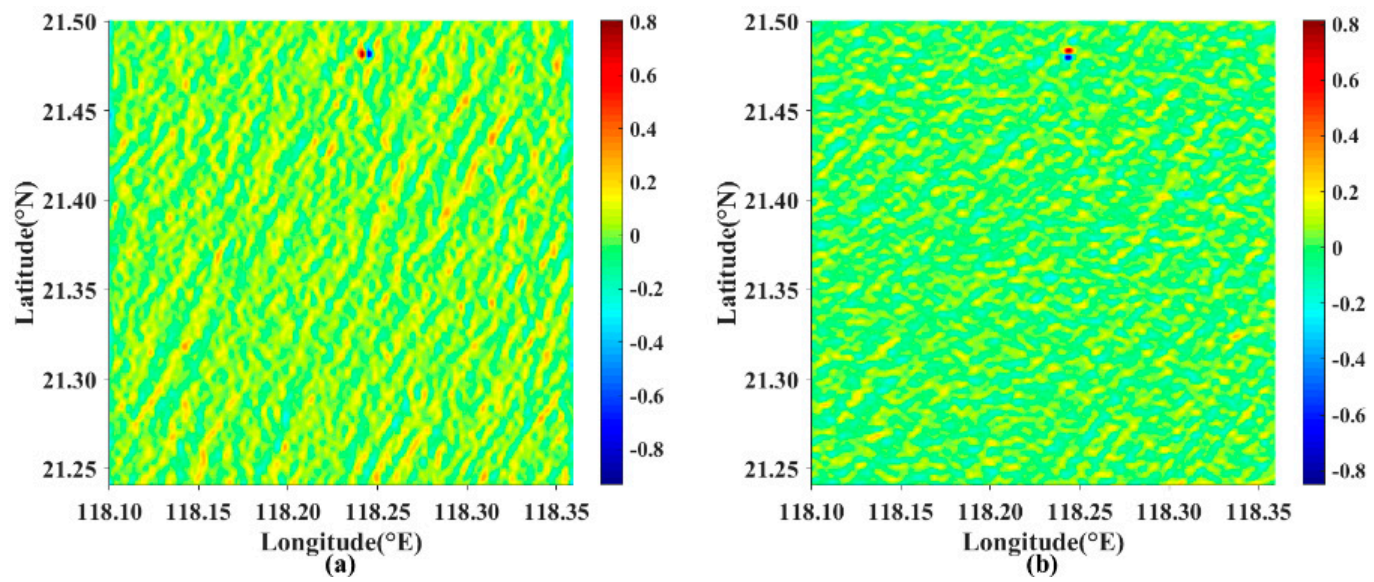

Figure 4. Computed $g_{x}(\mathbf{a})$ and $g_{y}(\mathbf{b})$ of the SAR image in Figure $2 \mathrm{~b}$.

\section{Data Sets}

\subsection{SAR Data}

The wind streaks do not appear in all regions of the full-frame SAR image, so we find the wind streak patterns in a SAR image and apply the retrieval methods to the sub-images at these locations. After that, we can validate the retrieval results against the interpolating wind directions at these locations. To compare the retrieved results of different methods with the CCMP products on $0.25^{\circ}$ grid, we test all methods over the $30 \mathrm{~km}$ by $30 \mathrm{~km}$ sub-images, uniformly. Finally, we acquired 62 Envisat ASAR sub-images between 2004 and 2011. Most of the images are located between $15^{\circ} \mathrm{N}$ to $35^{\circ} \mathrm{N}$ and $113^{\circ} \mathrm{E}$ to $129^{\circ} \mathrm{E}$. ASAR operates in the $\mathrm{C}$ band in a wide variety of acquisition modes. The incidence angles of ASAR range from $15^{\circ}$ to $45^{\circ}$. The ASAR images used here are in VV polarization with a spatial resolution of $75 \mathrm{~m}$ by $75 \mathrm{~m}$ in range and azimuth. Geometric calibrations are carried out by the Next ESA SAR Toolbox provided by the European Space Agency (ESA). The distribution of wind directions of all ASAR images used in the study is shown in Figure 5. The radius of each fan indicates the amount of wind directions in each interval.

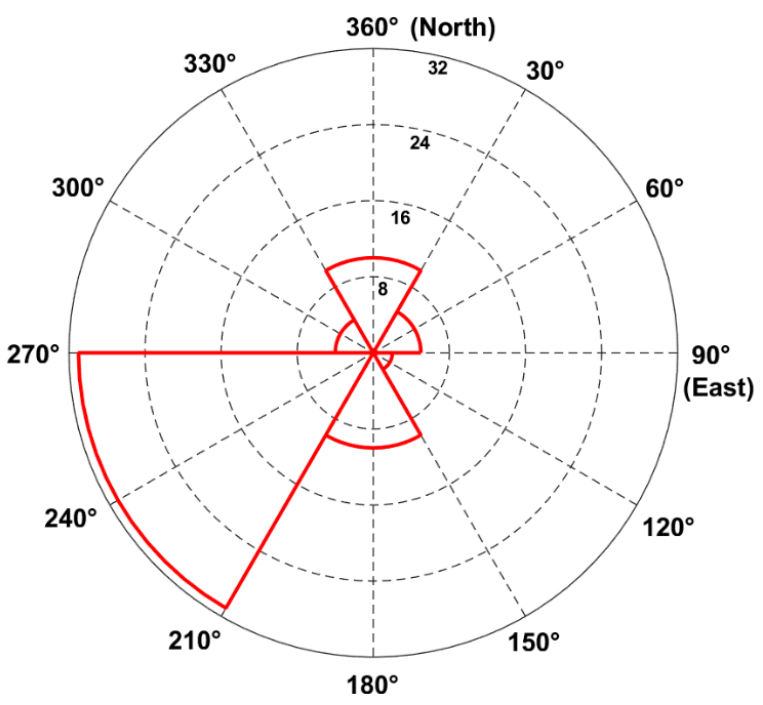

Figure 5. The distribution of wind directions of the images. 


\subsection{ECMWF Data}

The ERA-Interim is the latest global atmospheric reanalysis produced by ECMWF. The ERA-Interim reanalysis is produced with data assimilation, advancing forward using 12-hourly analysis cycles in time. In each cycle, available observations and prior information from a forecast model are combined to obtain the evolving state of the global atmosphere and its underlying surface. Thus, the data assimilation produces 6-hourly products at different spatial resolutions, which include various kinds of global surface parameters including $u$ and $v$ wind components at $10 \mathrm{~m}$ height [42]. It covers a long period starting in 1979 and continues updating in near real time. The detailed method for extracting the wind direction of a specific SAR image from the ECMWF data $\left(0.25^{\circ}\right)$ is as follows. First, the nearest location is found by the latitude and longitude of the center of the SAR image. Then, the $u$ and $v$ components at imaging time are obtained by interpolating the reanalysis data of the nearest two times. At last, the wind direction is computed by using the $u$ and $v$ components from interpolation.

\subsection{CCMP Data}

The wind directions from the CCMP wind product are used for validation. The CCMP data set combines cross-calibrated satellite microwave winds and instrument observations to produce high-resolution $\left(0.25^{\circ}\right)$ gridded analysis using a 4-dimensional variational analysis method with the ECWMF ERA-Interim Reanalysis model wind field as a background wind. Each daily data file contains three arrays of size 1440 (longitude) by 628 (from $78.375^{\circ} \mathrm{S}$ to $78.375^{\circ} \mathrm{N}$ ) by 4 (times of $0,6,12$ and 18 UTC) [9]. Two of the arrays are $u$ and $v$ wind components in meters per second at $10 \mathrm{~m}$ height. Another array in the file is the number of observations (satellites or buoys) used to derive the wind components for each grid and a number of observations value of zero means that the wind vector for this grid was obtained from the background field only as no satellite or moored buoy wind data were available. We prefer the data from satellite or instruments, so only the locations where the number of observations is more than zero are taken into consideration. The steps for extracting the wind direction of a specific SAR image from the CCMP data are similar to that from the ECMWF data.

\section{Results and Discussion}

To compare the results of different wind direction retrieval methods, the wind directions interpolated from ECMWF and CCMP data are used, respectively. For each $30 \mathrm{~km}$ by $30 \mathrm{~km}$ image, the interpolating wind direction was obtained by the nearest grid from the center of the image. Furthermore, the performance of the ILG and the other two methods for small regions (e.g., $7.5 \mathrm{~km}, 6 \mathrm{~km}$ and $3 \mathrm{~km}$ ) is discussed visually. We implemented the FFT and LG method based on algorithms described in the papers [34,38]. Therefore, we specify these methods as FFT-like method and LG-like method to suggest that they are our implementation of the original wind direction retrieval techniques, respectively.

\subsection{Comparison with ECMWF}

In this part, the wind directions interpolated from ECMWF data are used to compare the retrieved results of different methods. Figure 6 shows the comparison between the retrieved result of each method and ECMWF wind directions for all 62 images, and each dot represents the retrieved wind direction and the wind direction interpolated from ECMWF data. 


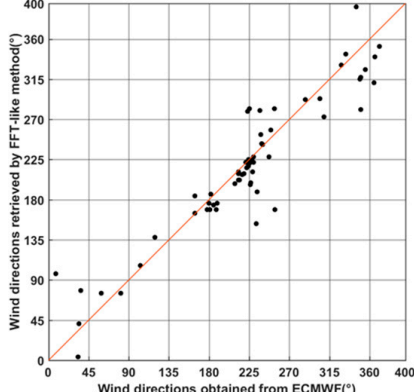

(a)

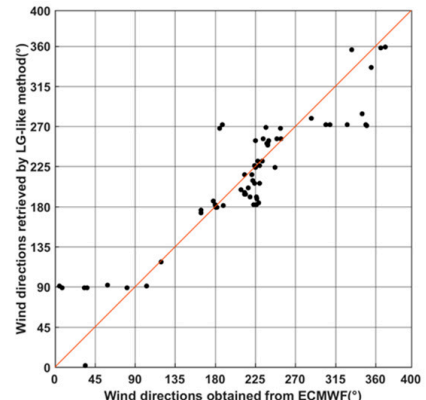

(b)

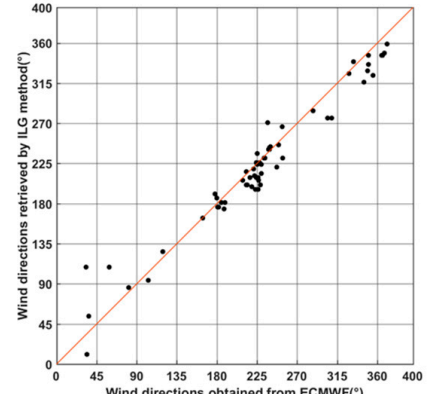

(c)

Figure 6. Comparison of wind directions between each retrieval method and ECMWF: (a) fast Fourier transform (FFT)-like method, (b) local gradients (LG)-like method, (c) ILG method.

From Figure 6, we can see that the wind directions retrieved by each method are obviously correlated with those from the ECMWF reanalysis data for these images. Table 1 shows the root-mean-square (RMS) error, correlation coefficient $(\mathrm{R})$ and $p$-value ( $p$-value demonstrates whether the two sets of data are correlated and the significance of correlation is larger when its value is smaller) of wind direction comparisons obtained by different retrieval methods and the ECMWF reanalysis data. In addition, if the absolute value of the angle difference between the retrieved direction and the ECMWF reanalysis data is bigger than $90^{\circ}$, the retrieved direction should add or subtract $360^{\circ}$ before computing these statistics, e.g., the retrieved direction is $2^{\circ}$ and the direction from ECMWF is $358^{\circ}$, so the retrieved direction should be converted to $362^{\circ}$. The process is similar for the computation with the CCMP data. We can see that the wind directions retrieved by the ILG method are closer to those of ECMWF reanalysis with the least RMS error of $21.57^{\circ}$ and the largest correlation coefficient of 0.9765. In addition, the $p$-value of ILG method is the smallest among the three methods. These statistics indicate that the retrieved results of the ILG method are the closest to the ECMWF reanalysis data among the three methods. By the way, the directional error of LG method in Koch's paper is $17.6^{\circ}$ for ERS-1/2 images, but there are no details about how to obtain the value. In fact, the validation results could be affected by several factors (e.g., the ground truth data, difference of different SAR sensors). In our study, we tested the different methods using ASAR images and the ECMWF (or CCMP) data, in order to compare their performances fairly. As a result, the statistics are different.

Table 1. The results of general statistics between wind directions obtained by different retrieval methods and ECMWF reanalysis data for 62 images.

\begin{tabular}{cccc}
\hline & FFT-Like Method & LG-Like Method & ILG Method \\
\hline $\operatorname{RMS}\left({ }^{\circ}\right)$ & 30.22 & 36.10 & 21.57 \\
R & 0.9344 & 0.9314 & 0.9765 \\
$p$-value & $1.38 \times 10^{-28}$ & $4.30 \times 10^{-25}$ & $1.03 \times 10^{-41}$ \\
\hline
\end{tabular}

\subsection{Comparison with CCMP}

We prefer the CCMP data with observations (satellites or buoys), and we matched up SAR images with the CCMP data which have observations. Finally, 22 images are matched. Figure 7 shows the comparison between the results of each method and CCMP wind directions for all matched images. 


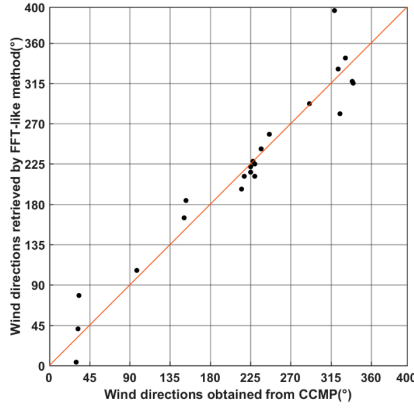

(a)

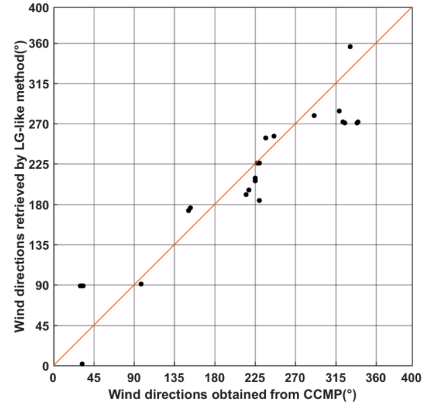

(b)

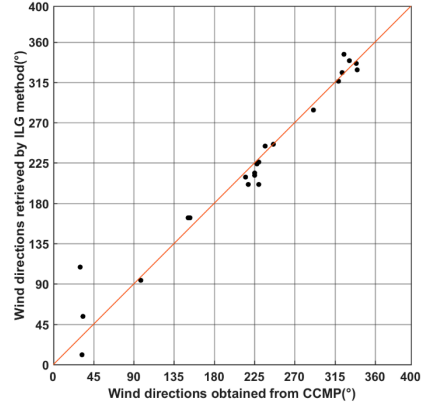

(c)

Figure 7. Comparison of wind directions between each retrieval method and CCMP: (a) FFT-like method, (b) LG-like method and (c) ILG method.

From Figure 7, we can see that the wind directions retrieved by each method show an obvious correlation with those from CCMP data for the matched images. Table 2 shows the results of statistics between wind directions obtained by each retrieval method and CCMP for these matched images. We can see that wind directions retrieved by the ILG method is the closest to the CCMP data with the least RMS error of $21.61^{\circ}$ and the largest correlation coefficient of 0.9771 . The $p$-value of ILG method is also the smallest. Consequently, the wind directions retrieved by the ILG method are the closest to CCMP data among the three methods.

Table 2. The results of general statistics between wind directions obtained by different retrieval methods and CCMP data for 22 images.

\begin{tabular}{cccc}
\hline & FFT-Like Method & LG-Like Method & ILG Method \\
\hline $\operatorname{RMS}\left(^{\circ}\right)$ & 25.41 & 36.65 & 21.61 \\
$\mathrm{R}$ & 0.9659 & 0.9413 & 0.9771 \\
$p$-value & $3.29 \times 10^{-13}$ & $6.92 \times 10^{-11}$ & $6.55 \times 10^{-15}$ \\
\hline
\end{tabular}

\subsection{Discussion}

The wind directions discussed above are retrieved from $30 \mathrm{~km}$ by $30 \mathrm{~km}$ images with a spatial resolution of $75 \mathrm{~m}$ in both range and azimuth. To test the performance of the ILG method in small regions, wind directions of the example image in Figure $2 \mathrm{~b}$ are computed on $7.5 \mathrm{~km}, 6 \mathrm{~km}$ and $3 \mathrm{~km}$ grids, respectively. The other two retrieval methods are also carried out on the same grids. Figure 8 shows the results of the three retrieval methods of the image on different grids. Yellow, magenta and cyan arrows indicate wind directions retrieved by the FFT-like, LG-like and ILG methods, respectively. The general wind direction of the image is from upper right to lower left. On the three kinds of grids in Figure 8, the retrieved results of the FFT-like method does not work well, and the retrieved results of the LG-like and ILG methods agree well with the streaks in the most areas. However, the retrieved results of the ILG method show better agreement with the streaks than that of the ILG method on the grids where the retrieved results of the two methods differ, visually. Another ASAR image acquired at 00:45, 25 March 2005 in VV polarization with a spatial resolution of $75 \mathrm{~m}$ was also tested. The image was along the coastal area of Iwate Prefecture, Japan. Figure 9 shows the image and the retrieved results of the three methods on $7.5 \mathrm{~km}, 6 \mathrm{~km}$ and $3 \mathrm{~km}$ grids, respectively. Yellow, magenta and cyan arrows indicate the wind directions retrieved by the FFT-like method, LG-like and ILG methods, respectively. From the SAR image, it can be seen that the general wind blows from upper left to lower right. From Figure 9a-c, we can see that the retrieved wind directions of the LG-like and ILG methods show better agreement with the streaks in most regions than the results of the FFT-like method. The retrieved wind directions of the ILG method still agree better with the general wind direction than the other two methods at the bottom of the image with the influence of the land. 


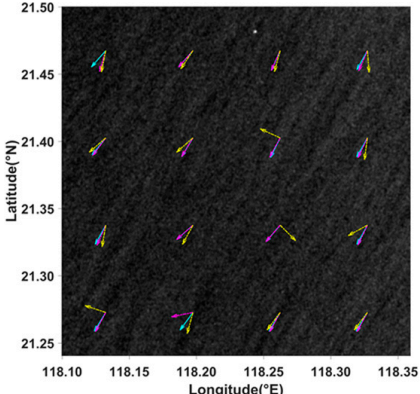

(a)

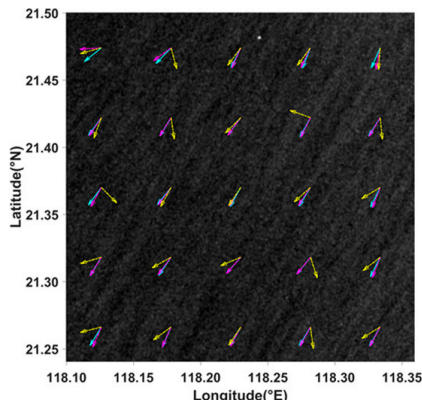

(b)

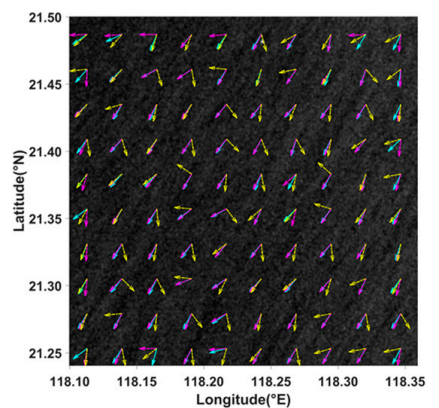

(c)

Figure 8. Wind directions retrieved by the three methods from $75 \mathrm{~m}$ pixels on different grids of the ASAR image in Figure 2b: (a) $7.5 \mathrm{~km}$ grid, (b) $6 \mathrm{~km}$ grid, (c) $3 \mathrm{~km}$ grid. Yellow, magenta and cyan arrows indicate wind directions retrieved by the FFT-like, LG-like and ILG methods, respectively.

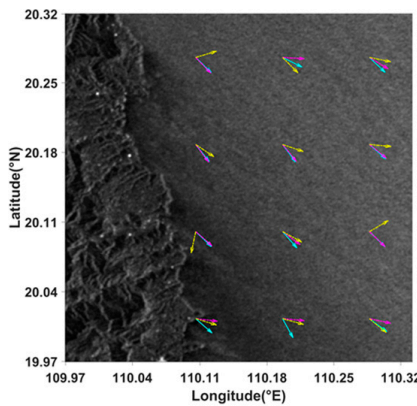

(a)

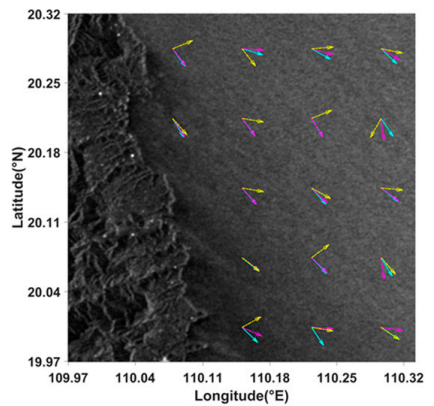

(b)

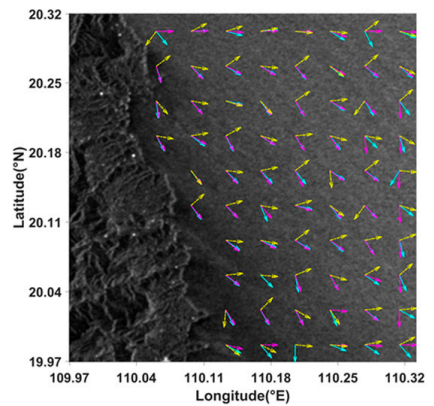

(c)

Figure 9. Wind directions retrieved by the three methods on different grids of the ASAR image acquired at 00:45, 25 March 2005 in VV polarization: (a) $7.5 \mathrm{~km}$ grid, (b) $6 \mathrm{~km}$ grid, (c) $3 \mathrm{~km}$ grid. Yellow, magenta and cyan arrows indicate wind directions retrieved by the FFT-like, LG-like and ILG methods, respectively.

We have tried to match all the ASAR sub-images we have with buoys, but none are matched. However, a Radarsat-2 image is matched with the buoy (46073) of National Data Buoy Center (NDBC). The Radarsat-2 image was acquired at 17:58, 11 February 2011 in VV polarization, and it was reduced to $80 \mathrm{~m}$ by $80 \mathrm{~m}$. A region ( $32 \mathrm{~km}$ by $32 \mathrm{~km}$ ) where the wind streaks are visible was cut out to perform the test. Figure 10 shows the results of the three retrieval methods of the sub-image on different grids $(8 \mathrm{~km}, 6.4 \mathrm{~km}$ and $3.2 \mathrm{~km})$, and the buoy is marked as a green dot in the SAR image. Again, the yellow, magenta and cyan arrows indicate wind directions retrieved by the FFT-like, LG-like and ILG methods, respectively. The wind direction $\left(263^{\circ}\right)$ measured by the buoy is indicated by the green arrow. From Figure 10, it can be seen that the retrieved wind directions of the LG-like and ILG methods agree better with the wind direction measured by the buoy than the FFT-like method. From Figure 10c, it can be seen that the retrieved results of the LG-like and ILG methods agree with each other in most regions, but the retrieved results of the ILG method show better agreement with the streaks than that of the LG method, especially at the edges of the SAR image. 


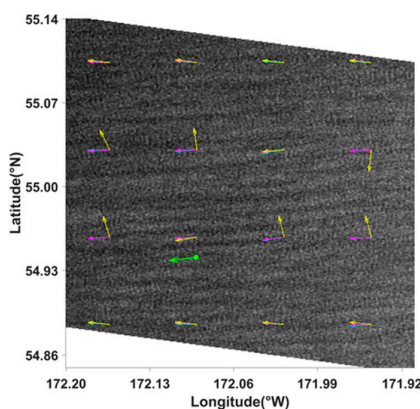

(a)

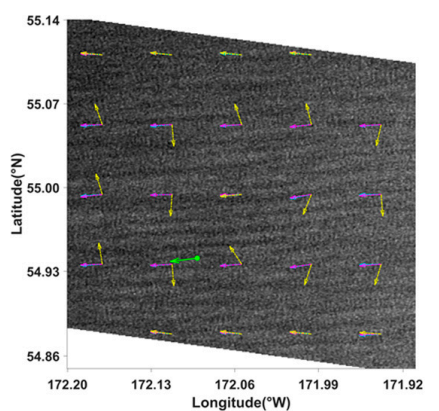

(b)

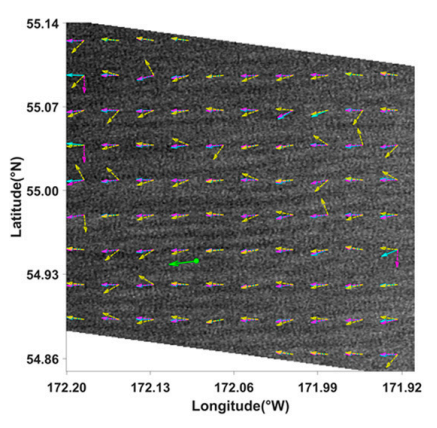

(c)

Figure 10. Wind directions retrieved by the three methods on different grids of the Radarsat- 2 image acquired at 17:58, 11 February 2011 in VV polarization: (a) $8 \mathrm{~km}$ grid, (b) $6.4 \mathrm{~km}$ grid, (c) $3.2 \mathrm{~km}$ grid. Yellow, magenta and cyan arrows indicate wind directions retrieved by the FFT-like, LG-like and ILG methods, respectively. The buoy (46073) is marked as the green dot and the in situ measurement is indicated by the green arrow.

\section{Sensitivities to Different Noise}

In the conventional LG method, the local gradients are computed using difference operators (e.g., Sobel operators), which is easily affected by noise. However, the ILG method does not need any difference approximation. Therefore, the sensitivities of the three retrieval methods to different types of noise are analyzed, including the salt-and-pepper noise, the additive noise and the multiplicative noise. It is assumed that there is no noise in the original SAR images before they are corrupted by the noise in the tests. Figure 11 shows the image corrupted by different noise, taking the image in Figure $2 \mathrm{~b}$ as an example. For a specific kind of noise with a specific intensity, the RMS of a specific method was calculated from the differences between the retrieved results of the 62 corrupted images by the method and those interpolated from the ECMWF data. After we calculate the RMS differences of the method for different intensity, we get the RMS curve of the method with respect to intensity for the kind of noise. The RMS curves are used to compare the sensitivity of each method. The same procedure was done for the 22 images matched with CCMP data.

\subsection{Salt-and-Pepper Noise}

Figure 12 shows the RMS differences between each of the three retrieval methods and interpolating wind directions of ECMWF or CCMP data when the salt-and-pepper noise exists in each image with different intensities. The intensity of the salt-and-pepper noise is decided by the percentage of contaminated pixels in the image. It is obvious that the quality of wind direction retrieval roughly decreases with the increase of noise level. The curve of the ILG method is below the curves of the other two methods in Figure 12a,b. It indicates that the ILG method can achieve better retrieved results than the other two methods comparing with ECMWF (or CCMP) data when the salt-and-pepper noise exists. 

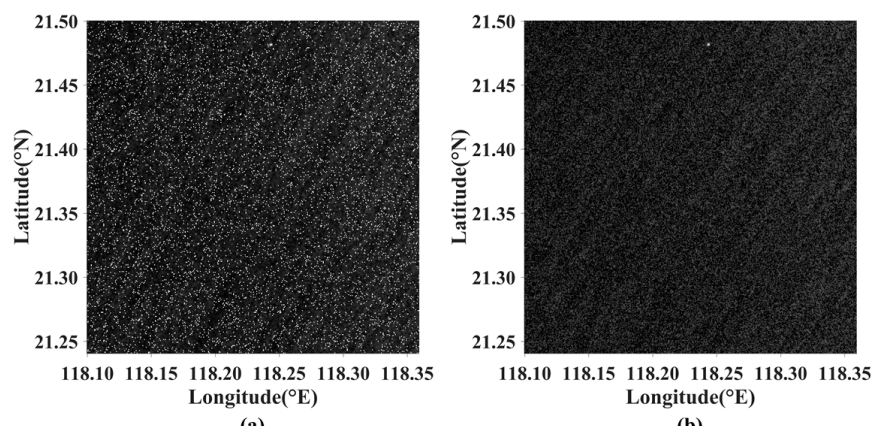

(a)

(b)
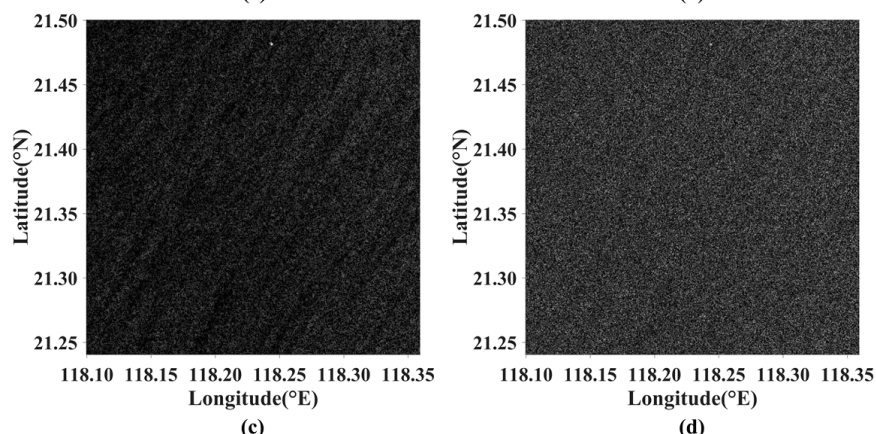

(d)

Figure 11. The image in Figure $2 b$ is corrupted by each kind of noise: (a) salt-and-pepper noise (10\% of contaminated pixels), (b) additive noise (SNR is $-10 \mathrm{~dB}$ ), (c) multiplicative noise (variance of $\mathrm{n}^{\prime}$ is 0.4 ), (d) speckle noise.

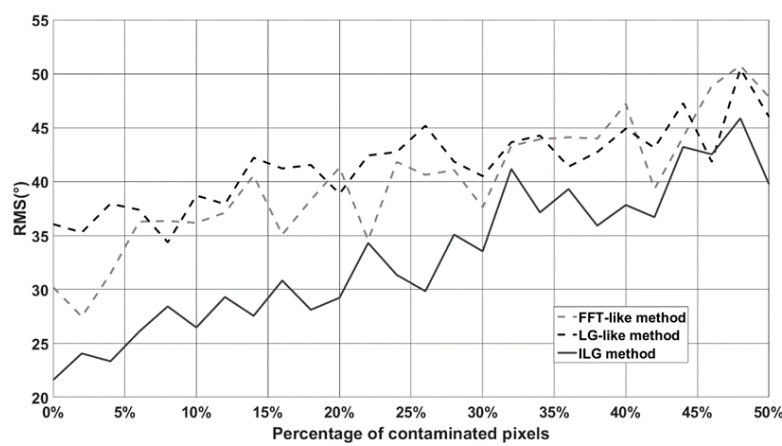

(a)

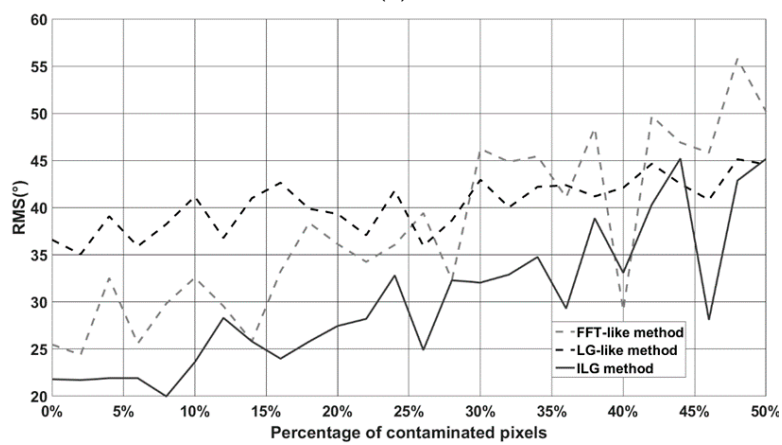

(b)

Figure 12. Root-mean-square (RMS) differences between the retrieved results of each method and the interpolating wind directions when the salt-and-pepper noise exists with different intensity: (a) ECMWF and (b) CCMP. 


\subsection{Additive Noise}

The additive noise is added to the image $I$ using the equation

$$
J=I+n
$$

where $n$ is the Gaussian white noise with zero mean and a specific variance. As mentioned above, it is assumed that there is no noise in the original SAR images before they are corrupted by the noise in the tests. Therefore, the signal-to-noise ratio (SNR) of an image corrupted by noise can be considered as the ratio of the variance of the original image and the variance of $n$. The intensity of the noise is lower when the SNR is larger. Figure 13 shows the RMS differences between the results of the three retrieval methods and interpolating wind directions when the images are corrupted by the additive noise with different SNR. We can find that all curves decline with increasing SNR, roughly. Furthermore, the ILG method can achieve better retrieved results than the other two methods comparing with ECMWF (or CCMP) data when the additive noise is present.

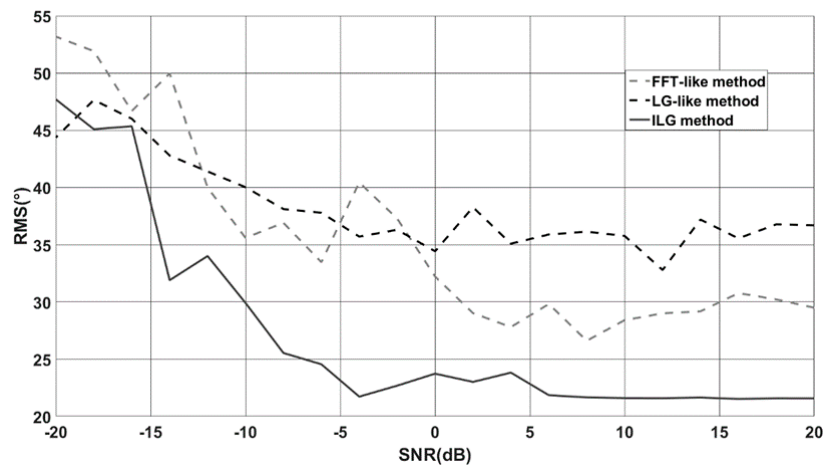

(a)

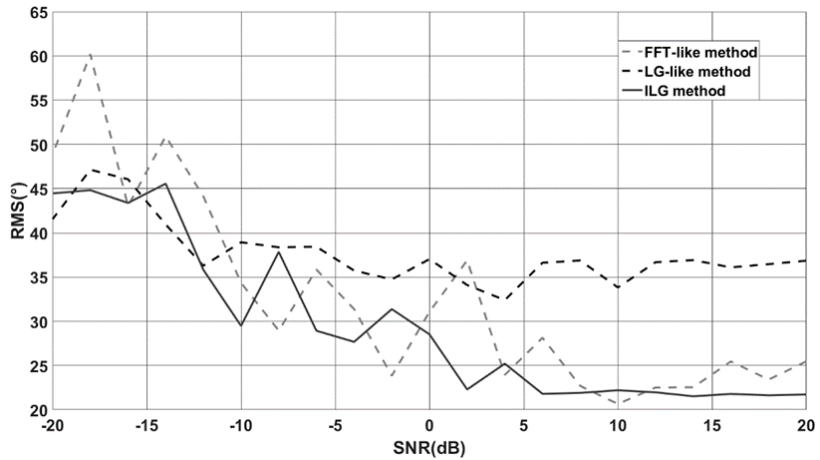

(b)

Figure 13. RMS differences between the retrieved results of each method and the interpolating wind directions when the additive noise exists with a different signal-to-noise ratio (SNR): (a) ECMWF and (b) CCMP.

\subsection{Multiplicative Noise}

The multiplicative noise is added to the image $I$ using the equation

$$
J=(1+n) I
$$

where $n$ is uniformly distributed random noise with zero mean and a given variance. The multiplicative noise intensity is decided by the given variance. The noise intensity increases with the increase of the variance. Figure 14 shows the RMS differences between the results of the three retrieval methods and interpolating wind directions while the multiplicative noise changes with different variance. 
The retrieved results of the three methods become worse roughly while the noise becomes greater. However, the ILG method always gives better retrieved results than the other two methods when the multiplicative noise is present comparing with ECMWF (or CCMP) data.

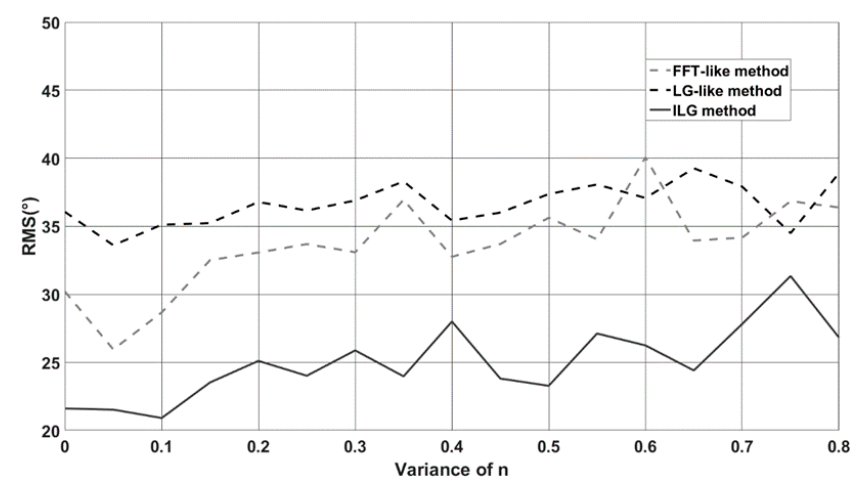

(a)

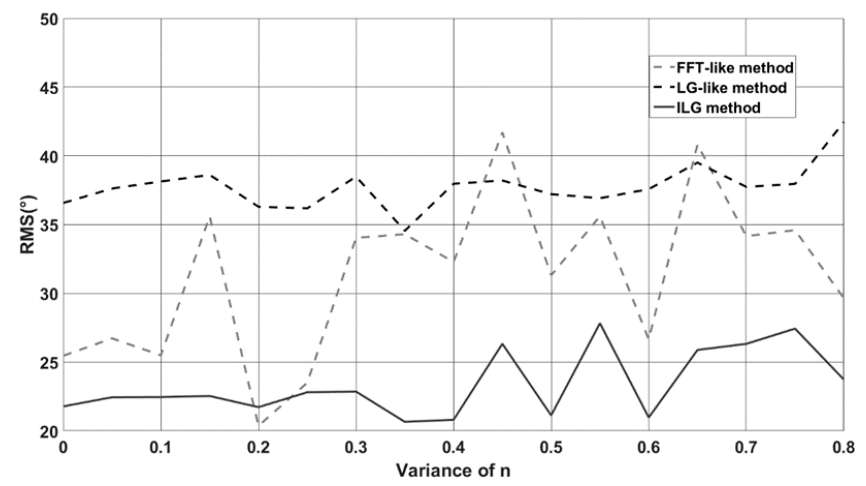

(b)

Figure 14. RMS differences between the retrieved results of each method and the interpolating wind directions when the multiplicative noise exists with different variance: (a) ECMWF and (b) CCMP.

The speckle noise is a kind of multiplicative noise as well, but the equation used to add the speckle noise to the image $I$ is a little different from Equation (12), which can be described as:

$$
J=n I
$$

where $n$ is the noise with an exponential distribution whose expectation and variance are 1 , as the SAR intensity images are corrupted. The RMS difference of each method between retrieved results of the images corrupted by this noise and wind directions obtained from the ECMWF (or CCMP) data is shown in Table 3. From Table 3, it can be seen that the retrieved results of the corrupted images of each method become worse than the results in Tables 1 and 2, as the speckle noise affects the retrieved results. However, the retrieved results of the corrupted images by the ILG method are better than the other two methods comparing with ECMWF (or CCMP) data when the speckle noise exists.

Table 3. The RMS differences between retrieved results of each method of the images corrupted by the speckle noise and wind directions obtained from the ECMWF (or CCMP) data.

\begin{tabular}{cccc}
\hline & FFT-Like Method & LG-Like Method & ILG Method \\
\hline ECMWF & $46.73^{\circ}$ & $45.29^{\circ}$ & $37.06^{\circ}$ \\
CCMP & $46.39^{\circ}$ & $42.14^{\circ}$ & $37.19^{\circ}$ \\
\hline
\end{tabular}


Form the analysis above, it can be found that the sensitivity of the ILG method to each kind of noise is lower than the other two methods.

\section{Conclusions}

In this study, the conventional LG method was improved by using the new approach to compute the local gradients. With the new approach, we can avoid the difference approximation which can be easily affected by noise. Comparing with ECMWF and CCMP data, we found that the RMS difference of the ILG method is smaller (about $15^{\circ}$ ) than the FFT-like and LG-like methods. The correlation coefficient of ILG method is the largest (about 0.98) among the three methods, suggesting that its retrieved results fit the ECMWF and CCMP data better than the other two methods. The ILG method could retrieve wind directions from small images (thus high resolution) with the tests on different grids, and the retrieved results of the ILG method on small grids (e.g., $3 \mathrm{~km})$ agree better with the wind streaks than the other two methods. When the SAR images containing both sea and land portions are processed, the ILG method still can work well. Furthermore, the sensitivity of the ILG method to different noises is lower than the other two methods, as the new method can avoid the use of difference approximation operators. All of these indicate that the ILG method is feasible.

Acknowledgments: This work is supported by the National Natural Science Foundation of China (Nos. 41676167, 41306192, 41476088, 41576174 and 41621064), the National Key R\&D Program of China (Nos. 2016YFC1401007, 2016YFC1401001 and 2016YFC1401005), and the Project of State Key Laboratory of Satellite Ocean Environment Dynamics, Second Institute of Oceanography (No. SOEDZZ1503), and the Key Research and Development Program of Hainan Province (No. ZDYF2017167). ASAR data is provided by ESA. The ERA-Interim reanalysis is produced by the European Centre for Medium-Range Weather Forecasts, and the data from the ECMWF is available at www.ecmwf.int. CCMP Version- 2.0 vector wind analyses, which are available at www.remss.com, are produced by the Remote Sensing Systems. The NDBC data is available at http:/ / www.ndbc.noaa.gov. I also want to thank Zuojun Yu for her suggestions on my English expressions. The views, opinions, and findings contained in this report are those of the authors and should not be construed as an official NOAA or U.S. Government position, policy or decision.

Author Contributions: Lizhang Zhou and Gang Zheng contributed the main idea and wrote the manuscript; Xiaofeng Li contributed to the design of the research and the article's organization; Jingsong Yang, Lin Ren and Peng Chen contributed to the collection the SAR images and data analysis; Huaguo Zhang and Xiulin Lou provided the data for comparative analysis and revised the manuscript. All authors have read and approved the submitted manuscript.

Conflicts of Interest: The authors declare no conflict of interest.

\section{References}

1. Friedman, K.S.; Sikora, T.D.; Pichel, W.G.; Clementecolón, P.; Hufford, G. Using spaceborne synthetic aperture radar to improve marine surface analyses. Weather Forecast. 2010, 16, 270-276. [CrossRef]

2. Von Ahn, J.M.; Sienkiewicz, J.M.; Chang, P.S. Operational impact of QuikSCAT winds at the NOAA ocean prediction center. Weather Forecast. 2006, 21, 523-539. [CrossRef]

3. Christiansen, M.B.; Koch, W.; Horstmann, J.; Hasager, C.B.; Nielsen, M. Wind resource assessment from C-band SAR. Remote Sens. Environ. 2006, 105, 68-81. [CrossRef]

4. Chang, R.; Zhu, R.; Badger, M.; Hasager, C.B.; Xing, X.; Jiang, Y. Offshore wind resources assessment from multiple satellite data and WRF modeling over south China sea. Remote Sens. 2015, 7, 467-487. [CrossRef]

5. Cavaleri, L.; Alves, J.H.; Ardhuin, F.; Babanin, A.; Banner, M.; Belibassakis, K.; Benoit, M.; Donelan, M.; Groeneweg, J.; Herbers, T.H.C. Wave modeling-The state of the art. Prog. Oceanogr. 2007, 74, 603-674. [CrossRef]

6. Sullivan, P.P.; Mcwilliams, J.C. Dynamics of winds and currents coupled to surface waves. Annu. Rev. Fluid Mech. 2009, 42, 19-42. [CrossRef]

7. Xu, Q.; Li, X.; Wei, Y.; Tang, Z.; Cheng, Y.; Pichel, W.G. Satellite observations and modeling of oil spill trajectories in the Bohai sea. Mar. Pollut. Bull. 2013, 71, 107-116. [CrossRef] [PubMed]

8. Cheng, Y.; Liu, B.; Li, X.; Nunziata, F. Monitoring of oil spill trajectories with Cosmo-SkyMed X-band SAR images and model simulation. IEEE J. Sel. Top. Appl. Earth Obs. Remote Sens. 2014, 7, 2895-2901. [CrossRef] 
9. Atlas, R.; Hoffman, R.N.; Ardizzone, J.; Leidner, S.M.; Jusem, J.C.; Smith, D.K.; Gombos, D. A cross-calibrated, multiplatform ocean surface wind velocity product for meteorological and oceanographic applications. Bull. Am. Meteorol. Soc. 2011, 92, 157-174. [CrossRef]

10. Salonen, K.; Niemelä, S.; Fortelius, C. Application of radar wind observations for low-level NWP wind forecast validation. J. Appl. Meteorol. 2011, 50, 1362-1371. [CrossRef]

11. Yang, X.; Li, X.; Zheng, Q.; Gu, X.F. Comparison of ocean-surface winds retrieved from QuikSCAT scatterometer and Radarsat-1 SAR in offshore waters of the US west coast. IEEE Geosci. Remote Sens. Lett. 2011, 8, 163-167. [CrossRef]

12. Wentz, F.J. Measurement of oceanic wind vector using satellite microwave radiometers. IEEE Trans. Geosci. Remote Sens. 1992, 30, 960-972. [CrossRef]

13. Naderi, F.M.; Freilich, M.H.; Long, D. Spaceborne radar measurement of wind velocity over the ocean-An overview of the NSCAT scatterometer system. Proc. IEEE 1991, 79, 850-866. [CrossRef]

14. Gerling, T.W. Structure of the surface wind field from the Seasat SAR. J. Geophys. Res. 1986, 91, $2308-2320$. [CrossRef]

15. Mastenbroek, K. High-resolution wind fields from ERS SAR. Earth Obs. Quart. 1998, 59, $20-22$.

16. Horstmann, J.; Koch, W. Ocean wind field retrieval using Envisat ASAR data. In Proceedings of the Geoscience and Remote Sensing Symposium, Toulouse, France, 21-25 July 2003; pp. 3102-3104.

17. Zhang, B.; Perrie, W.; He, Y. Wind speed retrieval from Radarsat-2 quad-polarization images using a new polarization ratio model. J. Geophys. Res. Atmos. 2011, 116, 1318-1323. [CrossRef]

18. Li, X. The first Sentinel-1 SAR image of a typhoon. Acta Ocean. Sin. 2015, 34, 1-2. [CrossRef]

19. Kim, T.S.; Park, K.A.; Li, X.; Hong, S. SAR-derived wind fields at the coastal region in the East/Japan sea and relation to coastal upwelling. Int. J. Remote Sens. 2014, 35, 3947-3965. [CrossRef]

20. Xuan, Z.; Yang, X.F.; Li, Z.W.; Yang, Y.; Bi, H.B.; Sheng, M.; Li, X.F. Estimation of tropical cyclone parameters and wind fields from SAR images. Sci. China Earth Sci. 2013, 56, 1977-1987.

21. Li, X.; Zhang, J.A.; Yang, X.; Pichel, W.G.; Demaria, M.; Long, D.; Li, Z. Tropical cyclone morphology from spaceborne synthetic aperture radar. Bull. Am. Meteorol. Soc. 2013, 94, 215. [CrossRef]

22. Li, X.; Pichel, W.G.; He, M.; Wu, S.Y. Observation of hurricane-generated ocean swell refraction at the Gulf Stream north wall with the Radarsat-1 synthetic aperture radar. IEEE Trans. Geosci. Remote Sens. 2002, 40, 2131-2142.

23. Friedman, K.S.; Li, X. Monitoring hurricanes over the ocean with wide swath SAR. Johns Hopkins Apl Tech. Dig. 2000, 21, 80-85.

24. Li, X.; Zheng, W.; Pichel, W.G.; Zou, C.Z.; Clemente-Colón, P. Coastal katabatic winds imaged by SAR. Geophys. Res. Lett. 2007, 34, 300-315. [CrossRef]

25. Li, X.; Zheng, W.; Yang, X.; Zhang, J.A.; Pichel, W.G.; Li, Z. Coexistence of atmospheric gravity waves and boundary layer rolls observed by SAR. J. Atmos. Sci. 2013, 70, 3448-3459. [CrossRef]

26. Liu, S.; Li, Z.; Yang, X.; Pichel William, G.; Yu, Y.; Zheng, Q.; Li, X. Atmospheric frontal gravity waves observed in satellite SAR images of the Bohai sea and Huanghai sea. Acta Ocean. Sin. 2010, 29, 35-43. [CrossRef]

27. Li, X.; Dong, C.; Clemente-Colón, P.; Pichel, W.G.; Friedman, K.S. Synthetic aperture radar observation of the sea surface imprints of upstream atmospheric solitons generated by flow impeded by an island. J. Geophys. Res. 2004, 109, 235-250. [CrossRef]

28. Chunchuzov, I.; Vachon, P.W.; Li, X. Analysis and modeling of atmospheric gravity waves observed in Radarsat SAR images. Remote Sens. Environ. 2000, 74, 343-361. [CrossRef]

29. Li, X.; Zheng, W.; Zou, C.Z.; Pichel, W.G. A SAR observation and numerical study on ocean surface imprints of atmospheric vortex streets. Sensors 2008, 8, 3321-3334. [CrossRef] [PubMed]

30. Li, X.; Clemente-Colón, P.; Pichel, W.G.; Vachon, P.W. Atmospheric vortex streets on a Radarsat SAR image. Geophys. Res. Lett. 2000, 27, 1655-1658. [CrossRef]

31. Li, X.; Yang, X.; Zheng, W.; Zhang, J.A. Synergistic use of satellite observations and numerical weather model to study atmospheric occluded fronts. IEEE Trans. Geosci. Remote Sens. 2015, 53, 1-11. [CrossRef]

32. Mouche, A.; Collard, F.; Chapron, B.; Dagestad, K.; Guitton, G.; Johannessen, J.A.; Kerbaol, V.; Hansen, M.W. On the use of Doppler shift for sea surface wind retrieval from SAR. IEEE Trans. Geosci. Remote Sens. 2012, 50, 2901-2909. [CrossRef] 
33. Wackerman, C.C.; Pichel, W.G.; Clemente-Colon, P. Automated estimation of wind vectors from SAR. In Proceedings of the 12th Annual Conference on Satellite Meteorology at the 83rd Annual Meteorological Association Meeting, Long Beach, CA, USA, 9-13 February 2003.

34. Koch, W. Directional analysis of SAR images aiming at wind direction. IEEE Trans. Geosci. Remote Sens. 2004, 42, 702-710. [CrossRef]

35. Zhang, G.; Perrie, W.; Li, X.; Zhang, J.A. A hurricane morphology and sea surface wind vector estimation model based on C-band cross-polarization SAR imagery. IEEE Trans. Geosci. Remote Sens. 2017, 55, 1-9. [CrossRef]

36. Zhang, B.; Perrie, W.; Vachon, P.W.; Li, X.; Pichel, W.G.; Guo, J.; He, Y. Ocean vector winds retrieval from C-band fully polarimetric SAR measurements. IEEE Trans. Geosci. Remote Sens. 2012, 50, 4252-4261. [CrossRef]

37. Liu, G.; Yang, X.; Li, X.; Zhang, B. A systematic comparison of the effect of polarization ratio models on sea surface wind retrieval from C-band synthetic aperture radar. IEEE J. Sel. Top. Appl. Earth Obs. Remote Sens. 2013, 6, 1100-1108. [CrossRef]

38. Wackerman, C.; Shuchman, R.; Fetterer, F. Estimation of wind speed and wind direction from ERS-1 imagery. In Proceedings of the Geoscience and Remote Sensing Symposium, Pasadena, CA, USA, 8-12 August 1994; pp. 1222-1224.

39. Yang, J.S.; Huang, W.G.; Zhou, C.B.; Bin, F.U.; Shi, A.Q.; Li, D.L. Coastal ocean surface wind retrieval from SAR imagery. J. Remote Sens. 2001, 5, 13-16.

40. Ulaby, F.T.; Moore, R.K.; Fung, A.K. Microwave Remote Sensing: Active and Passive, Volume 2-Radar Remote Sensing and Surface Scattering and Emission Theory; Addison-Wesley: Boston, MA, USA, 1982.

41. Wang, L.; Zhou, Y.; Liu, Y.; Zhang, M. Wind retrieval over the ocean using advanced synthetic aperture radar. In Proceedings of the International Conference on Geoinformatics, Shanghai, China, 24-26 June 2011; pp. 1-5.

42. Dee, D.P.; Uppala, S.M.; Simmons, A.J.; Berrisford, P.; Poli, P.; Kobayashi, S.; Andrae, U.; Balmaseda, M.A.; Balsamo, G.; Bauer, P. The ERA-interim reanalysis: Configuration and performance of the data assimilation system. Q. J. R. Meteorol. Soc. 2011, 137, 553-597. [CrossRef] 